\title{
Simulation of incompressible multiphase flows with complex geometry using etching multiblock method*
}

\author{
Haoran LIU, Kai MU, Hang DING ${ }^{\dagger}$ \\ Department of Modern Mechanics, University of Science and Technology of China, \\ Hefei 230027, China
}

\begin{abstract}
The incompressible two-phase flows are simulated using combination of an etching multiblock method and a diffuse interface (DI) model, particularly in the complex domain that can be decomposed into multiple rectangular subdomains. The etching multiblock method allows natural communications between the connected subdomains and the efficient parallel computation. The DI model can consider two-phase flows with a large density ratio, and simulate the flows with the moving contact line (MCL) when a geometric formulation of the MCL model is included. Therefore, combination of the etching method and the DI model has potential to deal with a variety of two-phase flows in industrial applications. The performance is examined through a series of numerical experiments. The convergence of the etching method is firstly tested by simulating single-phase flows past a square cylinder, and the method for the multiphase flow simulation is validated by investing drops dripping from a pore. The numerical results are compared with either those from other researchers or experimental data. Good agreement is achieved. The method is also used to investigate the impact of a droplet on a grooved substrate and droplet generation in flow focusing devices.
\end{abstract}

Key words etching multiblock method, complex geometry, multiphase flow, moving contact line (MCL), multiblock

Chinese Library Classification O242, O359, O363.2

2010 Mathematics Subject Classification 68U20, 76T10, 76M25, 76D45

\section{Introduction}

Interfacial flows in the domain with the complex geometry are often encountered in nature and industrial applications. These flows are usually difficult for numerical simulations, partially because they may involve with intricate physical phenomena, such as the moving contact line (MCL) at which the gas, liquid, and solid meet. Examples are the striding of insects on the water surface ${ }^{[1]}$ and the floating/sinking of solid objects ${ }^{[2]}$. It requires appropriate models for the interface and MCLs to perform the accurate computation. Moreover, the complex geometry also demands the generation of appropriate meshes, on which the equations of motion are solved. Traditionally, a body-fitted mesh is generated for this purpose, either structured ${ }^{[3]}$ or unstructured $^{[4]}$. Nowadays, because of its efficiency and simplicity, immerse boundary (IB) methods become more and more popular in dealing with flow problems in the presence of

* Received Jan. 25, 2016 / Revised Mar. 15, 2016

Project supported by the National Natural Science Foundation of China (No.11425210) and the

Fundamental Research Funds for the Central Universities (No. WK2090050025)

$\dagger$ Corresponding author, E-mail: hding@ustc.edu.cn 
complex geometry ${ }^{[5-7]}$. In the IB methods, the solid boundaries can be represented by a set of linked Lagrangian points, while the flows are solved on the Eulerian mesh. Since the solid boundaries generally do not coincide with the mesh lines, a feedback force is added in the momentum equation to account for the presence of the embedded solid boundaries. Recently, we combined the IB method ${ }^{[8]}$ with the diffuse interface (DI) model ${ }^{[9]}$, and successively simulated the flows with MCLs on curved substrates ${ }^{[10]}$. Despite of the success of IB methods, their accuracy depends on the way of representing the solid boundary, e.g., the use of delta function in the distribution of the feedback force makes this kind of IB methods essentially first-order accurate in modeling the solid boundaries.

Under certain circumstance, the complex geometry can be decomposed into several regular subdomains/blocks, of which each can be discretized by a structured mesh. Therefore, the governing equations can be solved in the individual block, and the communications between the blocks are exchanged by implementing appropriate boundary conditions (BCs). This way of dealing with the complex geometry is often referred to as the multiblock method. In the multiblock methods, we can still enjoy the merits of the structured mesh, such as easiness in constructing numerical schemes of high accuracy and simplicity in the parallel computation. Clearly, the main challenge in the multiblock approach lies in how to properly decompose the blocks and provide the BCs for each block. Wan et al. ${ }^{[11]}$ used block-structured grids with fictitious overlapping interfaces to decompose a complex computational geometry. Raspo ${ }^{[12]}$ solved the incompressible Navier-Stokes (NS) equations by a direct domain-decomposition method coupled with a Chebyshev collocation method. Abide and Viazzo ${ }^{[13]}$ used a direct non-overlapping multidomain method to simulate the incompressible viscous flows, such as von Karman vortex street behind a square cylinder. All these works only consider single-phase flows. This fact motivates us to examine the performance of the multiblock method in simulating multiphase flows, particularly in the presence of MCLs.

In the present study, we use an etching multiblock method coupled with the DI model to investigate incompressible multiphase flows with complex regular geometries. The etching multiblock method defines a regular domain as the global domain, and marks all the embedded solid boundaries. As a result, the blocks can be considered as the patches on a global structured mesh, which naturally establishes the connections between the blocks. The fluid-fluid interface is represented by a DI model ${ }^{[14]}$. This model replaces the mathematically sharp interface by a DI with the finite thickness, where the fluids are mixed to some extent. The DI model allows for the simulation of two-phase flows with the large density ratio ${ }^{[9]}$, and has been used to a variety of flow problems, such as vesicle dynamics ${ }^{[15]}$, Hele-Shaw flows ${ }^{[16]}$, and head-on droplet collision $^{[17]}$. We choose the geometric formulation of a DI model to model the motion of contact line on the substrate ${ }^{[18]}$. This model has been used to many two-phase flows in the presence of MCLs, including droplet spreading ${ }^{[19]}$, sliding of a three-dimensional droplet on a wall ${ }^{[20-21]}$, and ejection of satellite droplet in a rapid spreading ${ }^{[22]}$.

We present the modeling and numerical methods in Section 2, including the governing equations, MCL model, etching multiblock method, and BCs. A variety of numerical experiments are performed to validate the method in Section 3. We first simulate the flow past a square cylinder for the convergence study. Then, we investigate the drops dripping from a pore for code validation, and quantitatively compare the numerical results with experimental observations. We also investigate a drop impacting on a grooved substrate in Subsection 3.3 and droplet generation in flow focusing in Subsection 3.4.

\section{Methodology}

\subsection{DI model}

We investigate here the flows of two incompressible immiscible liquids (A and B) and use a DI method $^{[9]}$ to model the interface between the two liquids. In the DI method, the mathematically 
sharp interface is replaced by a DI with the finite thickness of a length scale $\epsilon$. The DI is represented by the volume fraction $C$ of the fluid $\mathrm{A}(0 \leqslant C \leqslant 1)$. Consequently, the time evolution of the interface can be governed by the convective Cahn-Hilliard $(\mathrm{CH})$ equation,

$$
\frac{\partial C}{\partial t}+\nabla \cdot(\boldsymbol{u} C)=\frac{1}{P e} \nabla^{2} \psi
$$

where $\boldsymbol{u}=(u, v)$ is the flow velocity, and $\psi$ is the chemical potential defined by

$$
\psi=\phi^{\prime}(C)-C n^{2} \nabla^{2} C,
$$

in which $u$ and $v$ are the velocity components in the $x$ - and $y$-directions, respectively, $\phi(C)=$ $C^{2}(1-C)^{2} / 4$ is the bulk energy density, the Cahn number $C n=\epsilon / L$ is the dimensionless measure of the thickness of the DI, and $L$ is the characteristic length. $P e$ is the Péclet number that represents the relative importance of the convective fluxes $(\boldsymbol{u} C)$ to the diffusive fluxes $(\nabla \psi)$. According to Ref. [9], we choose $P e=1 / C n$ so that the DI would approach the sharp interface limit with the mesh refinement.

A geometric formulation of wetting condition ${ }^{[18]}$ is used here to model the MCL, and serves as the $\mathrm{BC}$ for $C$ at a solid substrate. In the geometric formulation, the local variation of $C$ along the normal direction to the solid substrate should satisfy

$$
\boldsymbol{n} \cdot \nabla C=-|\boldsymbol{\tau} \cdot \nabla C| \cos \theta_{\mathrm{s}} / \sin \theta_{\mathrm{s}},
$$

where $\boldsymbol{n}$ and $\boldsymbol{\tau}$ are the normal vector and the tangential vector of the solid boundary, respectively, and $\theta_{\mathrm{s}}$ is the microscale contact angle.

\subsection{NS equations}

The equations of motion for the fluids are the dimensionless NS equations and continuity equation,

$$
\begin{aligned}
& \rho\left(\frac{\partial \boldsymbol{u}}{\partial t}+\boldsymbol{u} \cdot \nabla \boldsymbol{u}\right)=-\nabla p+\frac{1}{R e} \nabla \cdot\left(\mu\left(\nabla \boldsymbol{u}+\nabla \boldsymbol{u}^{\mathrm{T}}\right)\right)+\frac{\boldsymbol{f}_{\mathrm{s}}}{W e}-\frac{\rho}{F r} \boldsymbol{j}, \\
& \nabla \cdot \boldsymbol{u}=0
\end{aligned}
$$

where $\boldsymbol{j}$ denotes the unit vector in the vertical direction, and $\boldsymbol{f}_{\mathrm{s}}$ is the surface tension force,

$$
\boldsymbol{f}_{\mathrm{s}}=6 \sqrt{2} \psi \nabla C / C n
$$

The dimensionless averaged density $\rho$ and viscosity $\mu$ are defined as

$$
\begin{aligned}
& \rho=C+\rho_{\mathrm{B}}(1-C) / \rho_{\mathrm{A}}, \\
& \mu=C+\mu_{\mathrm{B}}(1-C) / \mu_{\mathrm{A}},
\end{aligned}
$$

where the subscripts $\mathrm{A}$ and $\mathrm{B}$ denote the liquids $\mathrm{A}$ and $\mathrm{B}$, respectively. The dimensionless group includes the Reynolds number $R e=\rho_{\mathrm{A}} U L / \mu_{\mathrm{A}}$, the Weber number $W e=\rho_{\mathrm{A}} U^{2} L / \sigma$, and the Froude number $F r=U^{2} /(g L)$, where $\sigma$ is the surface tension coefficient, $g$ is the gravitational acceleration, and $L$ and $U$ are the characteristic length and velocity, respectively. We shall also refer to the Ohnesorge number $O h=\sqrt{W e} / R e$ and the Bond number $B o=\rho_{\mathrm{A}} L^{2} g / \sigma$ in the discussion. 


\subsection{Etching method and discretization}

We consider here to use an etching multiblock method in the simulation of incompressible viscous two-phase flows in a domain with the complex geometry, which can be decomposed into multiple rectangle subdomains (or blocks). For the convenience of coding, the computational domain is defined on a rectangle that contains the physical domain. In such a way, we can use a uniform Cartesian mesh to discretize the domain, and the boundaries of the physical domain coincide with the mesh lines. As a result, each flow variable such as $u, v, p$, and $C$ in the domain is stored in one array, and thus can be accessed in the same manner as on a structured mesh, regardless of the complex geometry involved. Therefore, we can adopt the discretization method on the structured mesh to discretize the governing equations.

In the present study, the spatial discretization of the governing equations is performed on a staggered grid, as shown in Fig. 1. All the scalar quantities such as the volume fraction, chemical potentials, and pressure are defined at the cell centers, while the vector quantities such as the velocity components are defined at the cell faces. Unless otherwise mentioned, the central finite difference schemes are used for the spatial discretization of the $\mathrm{CH}$ equation and the NS equations.

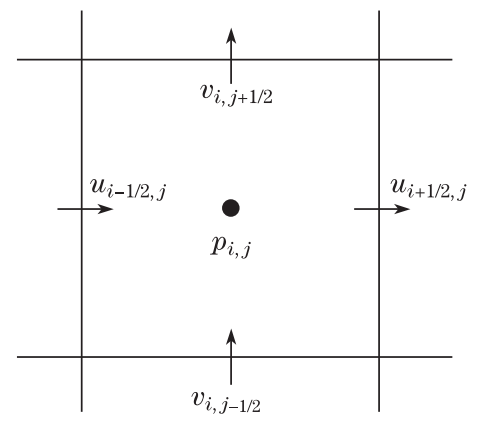

Fig. 1 Staggered grid, where all scalars such as volume fraction, chemical potential, and pressure are defined at cell centers $(\bullet)$, while vectors such as velocity components are defined at cell faces $(\rightarrow)$, and subscripts $(i, j)$ denote indices of cell

To solve the $\mathrm{CH}$ and NS equations in a temporally matched manner, the volume fraction $C$ field is defined at the time levels $t^{n-\frac{1}{2}}$, while the velocity $\boldsymbol{u}$ and the pressure $p$ are defined at $t^{n}(n=1,2,3, \cdots)$. Consequently, the $\mathrm{CH}$ equation (1) is discretized at the time level $n$ as follows:

$$
\begin{aligned}
\frac{C^{n+\frac{1}{2}}-C^{n-\frac{1}{2}}}{\Delta t}= & \left(\frac{3}{2} M\left(\boldsymbol{u}^{n}, C^{n-\frac{1}{2}}\right)-\frac{1}{2} M\left(\boldsymbol{u}^{n-1}, C^{n-\frac{3}{2}}\right)\right) \\
& -\frac{C n^{2}}{2 P e}\left(\nabla^{4} C^{n+\frac{1}{2}}+\nabla^{4} C^{n-\frac{1}{2}}\right)
\end{aligned}
$$

where we set the time step $\Delta t \sim h^{2}$ due to the explicit discretization of the second term in the next equation, and $h$ is the mesh space. We use the Adams-Bashforth and Crank-Nicolson schemes for the discretization of $M(\boldsymbol{u}, C)$ and $\nabla^{4} C$, respectively, where the term $M(\boldsymbol{u}, C)$ is defined as

$$
M(\boldsymbol{u}, C)=-\nabla \cdot(\boldsymbol{u} C)+\frac{1}{P e} \nabla^{2} \phi^{\prime}(C) .
$$

Note that the fluxes at the cell faces (the first term in Eq. (10)) are evaluated with a fifth-order weighted essentially non-oscillatory (WENO) scheme, using the local flow velocity to determine the upwind direction ${ }^{[9]}$. 
A standard projection method is used to couple the momentum equation with the continuity equation. The equations are discretized at the time level $\left(n+\frac{1}{2}\right)$ as follows:

$$
\begin{aligned}
\rho^{n+\frac{1}{2}}\left(\frac{\boldsymbol{u}^{*}-\boldsymbol{u}^{n}}{\Delta t}\right)= & \frac{1}{2 R e}\left(L\left(\boldsymbol{u}^{n+1}, \mu^{n+\frac{1}{2}}\right)+L\left(\boldsymbol{u}^{n}, \mu^{n+\frac{1}{2}}\right)\right)+\frac{f_{\mathrm{s}}^{n+\frac{1}{2}}}{W e}-\frac{\rho^{n+\frac{1}{2}}}{F r} \boldsymbol{j} \\
& -\rho^{n+\frac{1}{2}}\left(\frac{3}{2} H\left(\boldsymbol{u}^{n}\right)-\frac{1}{2} H\left(\boldsymbol{u}^{n-1}\right)\right),
\end{aligned}
$$

where we use the Crank-Nicolson scheme in the discretization of the viscous term $L=\nabla$. $\left(\mu^{n+\frac{1}{2}}\left(\nabla \boldsymbol{u}+\nabla \boldsymbol{u}^{\mathrm{T}}\right)\right)$, and the Adams-Bashforth scheme in the discretization of the advective term, $H=(\nabla \cdot \boldsymbol{u u})$. Note that $\rho^{n+\frac{1}{2}}, \mu^{n+\frac{1}{2}}$, and $\boldsymbol{f}_{\mathrm{s}}^{n+\frac{1}{2}}$ are functions of $C^{n+\frac{1}{2}}$.

The pressure $p^{n+1}$ is updated by solving a Poisson equation, i.e.,

$$
\nabla \cdot\left(\frac{\nabla p^{n+1}}{\rho^{n+\frac{1}{2}}}\right)=\frac{\nabla \cdot \boldsymbol{u}^{*}}{\Delta t}
$$

The velocity $\boldsymbol{u}^{n+1}$ is eventually made divergence-free after the pressure correction,

$$
\boldsymbol{u}^{n+1}=\boldsymbol{u}^{*}-\frac{\Delta t}{\rho^{n+\frac{1}{2}}} \nabla p^{n+1} .
$$

An over-relaxation iterative method is used to solve the Poisson equation in Eq. (12).

\subsection{BCs}

How to deal with BCs is the most important issue in the computation with the etching multiblock method. Here, we choose the case of drops dripping from a pore as an example to illustrate the solution procedure. The computational domain is shown in Fig. 2, in which the actual computational domain can be divided into two blocks, i.e., the rectangles $A H F E$ and $H B C G$, respectively. There are four kinds of flow BCs at the edges of these blocks, i.e., symmetric $(A H$ and $H B)$, inlet $(A E)$, outlet $(C B$ and $C G)$, and solid $(E F$ and $F G)$. There are also BCs particularly related to the etching multiblock method. For example, the edge $F H$ is considered as the block boundary, since it separates the blocks $a$ and $b$, although it is inside the computational domain. It is referred here to as the connection BC. A mixed $\mathrm{BC}$ is enforced at the boundary $G H$ of the block $b$, which consists of two edges $(G F$ and $F H)$ with different BCs.

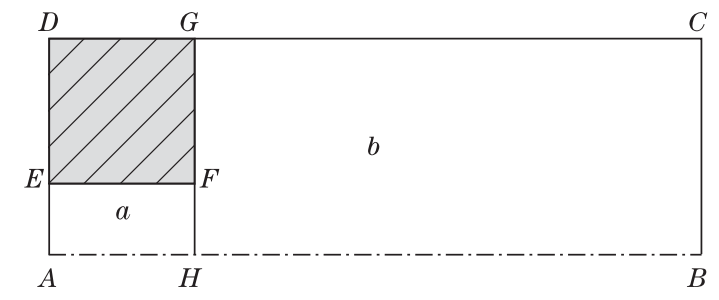

Fig. 2 Illustration of etching method, where computational domain is defined as rectangle $A B C D$ including solid wall $E D G F$, and actual computational domain can be decomposed into two blocks, $A H F E$ and $H B C G$

During the computation, the governing equations are solved in the two blocks one by one, along with the update of the BCs at the boundaries of each block. In the present study, we use one layer of ghost cells around each block to help the implementation of the BCs (see Fig. 3). In particular, the values of the flow variables at the ghost cells are updated according to the local BCs. 


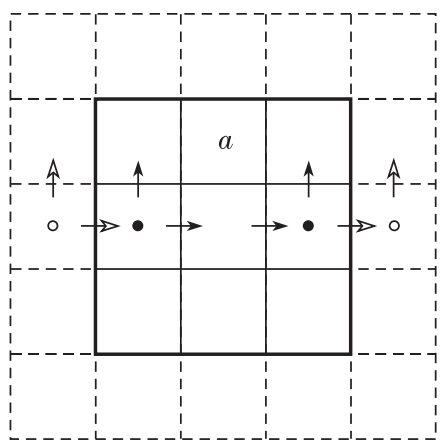

Fig. 3 Layer of ghost cells (o) around inner cells (

In the simulation of drops dripping from a pore, the BCs are specified as follows: at the left boundary (inlet), $u=u_{\text {in }}, v=0, \frac{\partial p}{\partial x}=0$, and $C=C_{\text {in }}$, where $u_{\text {in }}$ and $C_{\text {in }}$ are the prescribed horizontal velocity and the volume fraction at the inlet, respectively; at the right boundary (outlet), we use a characteristic BC, which is an approximation of the far-field BC, so that all the generated drops can pass smoothly through the boundary. Specially, the characteristic BCs can be expressed as

$$
\frac{\partial u}{\partial t}+U\left(\frac{\partial u}{\partial x}\right)=0, \quad \frac{\partial v}{\partial x}=0, \quad \frac{\partial p}{\partial x}=0, \quad \frac{\partial C}{\partial t}+U\left(\frac{\partial C}{\partial x}\right)=0 .
$$

At the bottom boundary, a symmetric BC is enforced, which can be expressed as

$$
\frac{\partial u}{\partial y}=0, \quad v=0, \quad \frac{\partial p}{\partial y}=0, \quad \frac{\partial C}{\partial y}=0 .
$$

At the solid wall, the no-slip BC is used for the velocity, and the geometric wetting condition is used for $C$, i.e.,

$$
\left\{\begin{array}{l}
u=0, \quad v=0, \quad \frac{\partial p}{\partial \boldsymbol{n}}=0, \\
\frac{\partial C}{\partial \boldsymbol{n}}=\left\{\begin{array}{c}
-\left(\frac{\partial C}{\partial \boldsymbol{\tau}}\right) \cot \theta_{\mathrm{s}}, \\
0, \quad \text { otherwise. }
\end{array}\right.
\end{array}\right.
$$

In the implementation of the connection $\mathrm{BC}$, there is no need to update the values at the ghost cells, which are essentially the inner cells. In this sense, we do not require any treatment at the connection BC. For the mixed BC, we only enforce the solid BC at the part of boundary at the solid wall, while leaving alone the part of boundary connected with other blocks.

The details of the discretized BCs are listed as follows:

(i) Solid BC

$$
\left\{\begin{array}{l}
u_{1 / 2, j}=0, \quad v_{0, j+1 / 2}=-v_{1, j+1 / 2}, \quad p_{0, j}=p_{1, j}, \\
C_{0, j}=\left\{\begin{array}{l}
C_{2, j}+\left|C_{1, j+1}-C_{1, j-1}\right| \cot \theta, \quad \text { if } \quad C_{1, j} \in[0.005,0.995], \\
C_{1, j}, \quad \text { otherwise }
\end{array}\right.
\end{array}\right.
$$

(ii) Symmetric BC

$$
u_{1 / 2, j}=0, \quad v_{0, j+1 / 2}=v_{1, j+1 / 2}, \quad p_{0, j}=p_{1, j}, \quad C_{0, j}=C_{1, j} .
$$

(iii) Inlet BC

$$
u_{1 / 2, j}=u_{\text {in }}, \quad v_{0, j+1 / 2}=0, \quad p_{0, j}=p_{1, j}, \quad C_{0, j}=C_{\mathrm{in}} .
$$


(iv) Characteristic BC

$$
\left\{\begin{array}{l}
u_{1 / 2, j}^{n+1}=u_{1 / 2, j}^{n}-\frac{\Delta t}{h} u_{1 / 2, j}^{n}\left(u_{3 / 2, j}^{n}-u_{1 / 2, j}^{n}\right), \\
v_{0, j+1 / 2}=v_{1, j+1 / 2}, \\
p_{0, j}=p_{1, j}, \\
C_{0, j}^{n+1 / 2}=C_{0, j}^{n-1 / 2}-\frac{\Delta t}{h} u_{1 / 2, j}^{n}\left(C_{1, j}^{n}-C_{0, j}^{n}\right),
\end{array}\right.
$$

where all the flow variables are defined in Fig. 4 , and the subscript $i=0$ denotes the ghost cells.

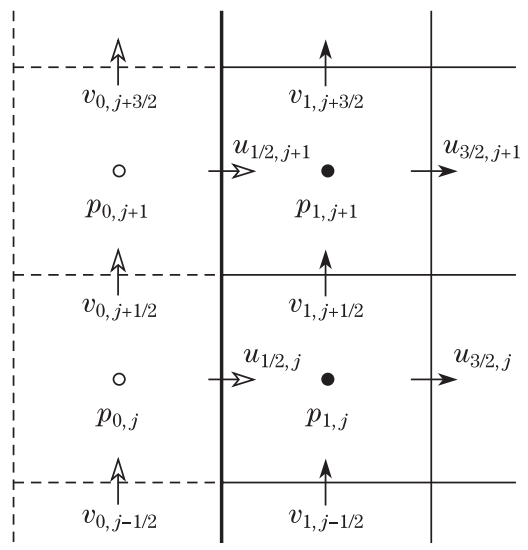

Fig. 4 Update of flow variables at ghost cells according to BCs at boundaries of block, where variables with subscript $i=0$ are supposed to be at ghost cells

For simplicity and convenience, we refer the solid $\mathrm{BC}$ to as $\mathrm{BC}_{1}$, and similarly, the symmetric $\mathrm{BC}\left(\mathrm{BC}_{2}\right)$, the inlet $\mathrm{BC}\left(\mathrm{BC}_{3}\right)$, the characteristic $\mathrm{BC}\left(\mathrm{BC}_{4}\right)$, the connection $\mathrm{BC}\left(\mathrm{BC}_{5}\right)$, and the mixed $\mathrm{BC}\left(\mathrm{BC}_{6}\right)$. In the following, we shall list the $\mathrm{BCs}$ for the block in a boundary sequence of left, bottom, right, and top. For example, for the block $a$ in Fig. 2, the BCs can be represented by $\left(\mathrm{BC}_{3}, \mathrm{BC}_{2}, \mathrm{BC}_{5}, \mathrm{BC}_{1}\right)$.

\section{Results and discussion}

In all the tests, the liquid-gas interface is represented by the contour $C_{\mathrm{L}}=0.5$, and the Cahn number is set to $C n=h$.

\subsection{Flow past square cylinder}

The convergence of the etching multiblock method is tested by simulating a two-dimensional single-phase flow past a square cylinder inside a channel. The numerical setup is shown in Fig. 5 , in which the computational domain of the size $8 D \times 50 D$ is split into four blocks $(a, b$, $c$, and $d)$, where $D$ is the side length of the square cylinder. The $\mathrm{BCs}$ are $\left(\mathrm{BC}_{3}, \mathrm{BC}_{1}, \mathrm{BC}_{6}\right.$, $\left.\mathrm{BC}_{1}\right)$ for the block $a,\left(\mathrm{BC}_{5}, \mathrm{BC}_{1}, \mathrm{BC}_{5}, \mathrm{BC}_{1}\right)$ for the blocks $b$ and $c$, and $\left(\mathrm{BC}_{6}, \mathrm{BC}_{1}, \mathrm{BC}_{4}\right.$, $\mathrm{BC}_{1}$ ) for the block $d$. It is assumed that the flow at the inlet is a fully developed laminar channel flow. Therefore, the prescribed velocity at the inlet $u_{\text {in }}$ has a parabolic velocity profile, $u_{\text {in }}=U_{\max }\left(-y^{2} /\left(16 D^{2}\right)+y /(2 D)\right)$, where $U_{\max }$ is the velocity in the middle of the inlet of the channel. The blockage ratio, i.e., the length of the cylinder side over the height of the channel, is 0.125 . 


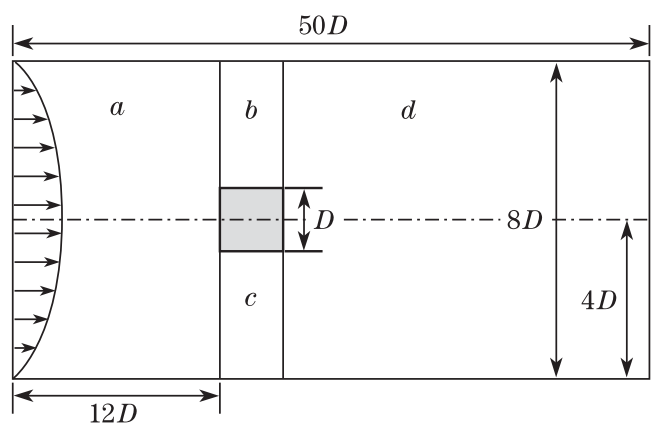

Fig. 5 Sketch of flow past square cylinder, where computational domain is decomposed into four blocks, i.e., blocks $a, b, c$, and $d$, respectively

We choose $D$ and $U_{\max }$ as the characteristic length and velocity, respectively. For the flow past a square cylinder, it is known that there exists a steady recirculating flow region at the downstream of the square cylinder at a relatively low $R e(0.5 \leqslant R e \leqslant 60)$. In contrast, the vortex shedding occurs at a relatively high $R e(60 \leqslant R e \leqslant 300)^{[23]}$. We select the cases at $R e=40$ and 100 to test the etching multiblock method. Figure 6 shows the numerical results in terms of streamlines. It is clear that steady flows are obtained at $R e=40$, and unsteady flows with vortex shedding occur at $R e=100$ (the time step $\Delta t=0.003$ and the mesh is $256 \times 1600)$, consistent with the prediction in Ref. [23]. To quantitatively validate the method, we make a comparison of our results with those from other researchers ${ }^{[3,23-24]}$ in Table 1, where EXP, FVM, LBA, and PDM represent the experiment, the finite-volume method, the latticeBoltzmann automata, and the projection decomposition method, respectively. In particular, we compare the length of the recirculating flow region $\left(L_{\mathrm{r}}\right)$ and the drag coefficient $\left(C_{\mathrm{d}}\right)$ at $R e=40$, and the averaged drag coefficient $\left(\bar{C}_{\mathrm{d}}\right)$ and the Strouhal number $S t\left(=f D / U_{\max }\right)$ at $R e=100$, where $f$ is the vortex shedding frequency. $C_{\mathrm{d}}$ is defined by

$$
C_{\mathrm{d}}=\frac{2 F_{x}}{\rho U_{\max }^{2} D},
$$

where $F_{x}$ is the drag force along the flow direction exerted by the surrounding fluid on the square cylinder. The present results on the successively refined meshes are listed in Table 1, i.e., $192 \times 1200,256 \times 1600$, and $320 \times 2000$, to test the convergence of the method. Clearly, the present results are in agreement with those from other researchers, and they are also converged with the successive mesh refinement.

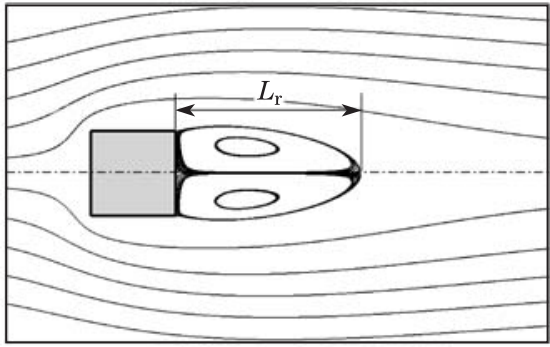

(a) $R \mathrm{e}=40$

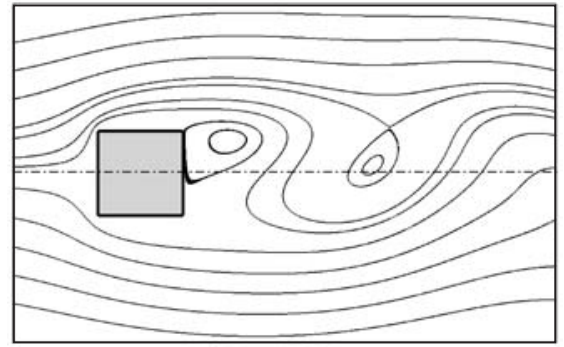

(b) $R \mathrm{e}=100$

Fig. 6 Numerical results of flow past square cylinder at (a) $R e=40$ and (b) $R e=100$ in terms of streamlines

\subsection{Drops dripping from pore}

Due to the effect of gravity, a drop attached to the ceiling could drip down if its volume is beyond a critical value ${ }^{[25]}$. This process exhibits many interesting phenomena, such as genera- 
tion of droplets, formation of a jet, and MCLs. We consider here the axisymmetric simulation of the dripping process of the water that leaks out of a pore. The density and viscosity ratios of the two liquids, water and air, are $\rho_{\mathrm{a}} / \rho_{\mathrm{w}}=0.001$ and $\mu_{\mathrm{a}} / \mu_{\mathrm{w}}=0.025$, respectively, where the subscripts a and w denote air and water, respectively. Figure 7 shows the numerical setup, in which the domain has a size of $1.5 D \times 9 D$, where $D$ is the diameter of the pore. The computational domain can be divided into two blocks $(a$ and $b$ ), and the former has a size of $0.5 D \times D$ and the latter is $1.5 D \times 8 D$. The $\mathrm{BCs}$ are $\left(\mathrm{BC}_{3}, \mathrm{BC}_{2}, \mathrm{BC}_{5}, \mathrm{BC}_{1}\right)$ for the block $a$ and $\left(B C_{6}, \mathrm{BC}_{2}, \mathrm{BC}_{4}, \mathrm{BC}_{4}\right)$ for the block $b$. The simulation is performed on a Cartesian mesh of $1800 \times 300$. We choose the liquid velocity $U$ on the entry boundary and the diameter of the pore $D$ as the characteristic velocity and length, respectively. The time step $\Delta t=0.0001$.

Table 1 Comparison of numerical results at $R e=40$ and 100

\begin{tabular}{|c|c|c|c|c|c|}
\hline \multirow{2}{*}{ Method } & \multirow{2}{*}{ Mesh } & \multicolumn{2}{|c|}{$R e=40$} & \multicolumn{2}{|c|}{$R e=100$} \\
\hline & & $C_{\mathrm{d}}$ & $L_{\mathrm{r}}$ & $\bar{C}_{\mathrm{d}}$ & $S t$ \\
\hline$\overline{\operatorname{EXP}^{[24]}}$ & - & - & - & - & $0.137 \pm 0.003$ \\
\hline $\mathrm{FVM}^{[23]}$ & $340 \times 560$ & 1.760 & 2.180 & 1.350 & 0.139 \\
\hline $\mathrm{LBA}^{[23]}$ & $320 \times 2000$ & 1.710 & 2.150 & 1.380 & 0.138 \\
\hline \multirow[t]{2}{*}{$\mathrm{PDM}^{[13]}$} & $1644 \times 1644$ & - & - & 1.390 & 0.143 \\
\hline & $320 \times 2000$ & 1.772 & 2.184 & 1.389 & 0.1379 \\
\hline \multirow[t]{2}{*}{ Present } & $256 \times 1600$ & 1.774 & 2.186 & 1.400 & 0.1379 \\
\hline & $192 \times 1200$ & 1.779 & 2.192 & 1.412 & 0.1371 \\
\hline
\end{tabular}

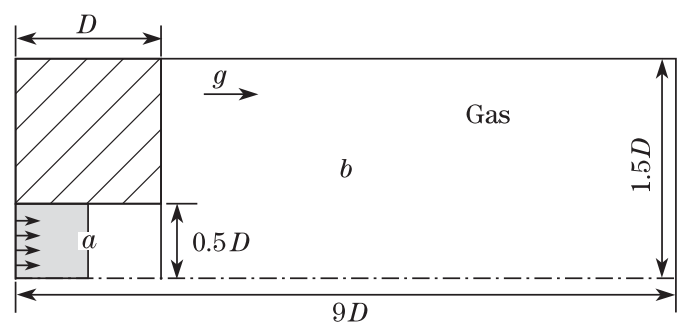

Fig. 7 Sketch of drops dripping from pore, where gray area shows initial space occupied by liquid, blank area is supposed to be filled with air, and direction of gravity is from left to right

We simulate two cases with the same $R e(=4.34), B o(=0.33)$, and wettability $\theta\left(=120^{\circ}\right)$ but different $W e: W e=0.16$ and 0.2 . All the dimensionless numbers are defined by the use of the properties of the water. In the simulation, the liquid that leaks out of the pore gradually accumulates at the end of the pore, and due to the surface tension force, a drop comes into being and grows with the time. When the drop becomes sufficiently large, a droplet breaks up from the drop and drips down under the influence of gravity. During the whole dynamic process, the contact lines are pinned at the corner of the pore, primarily due to the geometry-induced contact-angle hysteresis ${ }^{[26-27]}$. Figure 8 shows the snapshots of the periodic dripping process from the pore. It is interesting to see that the difference in $W e$ leads to different dripping modes, i.e., the single-droplet mode and the double-droplet mode. More details of the dripping process are shown in Fig. 9, in which the time interval between the successive generation of droplets $\left(t_{\mathrm{d}}\right)$ is presented as a function of the sequence number of the droplets $(N)$. Clearly, the dripping process results in a periodic generation of droplets, and the Strouhal number $S t\left(=f \sqrt{D_{\mathrm{d}} / g}\right.$, where $D_{\mathrm{d}}$ is the diameter of the generated droplet) is 19.2 for the single-droplet mode and 23.6 and 20.1 for the double-droplet mode. Subramani et al. ${ }^{[28]}$ conducted experiments with similar 
parameters, and they also found the two modes. Moreover, they observed that the volume of the generated droplet was 20.8 for the single-droplet mode, and our numerical result gives nearly the same value (20.7). For the double-droplet mode, the experimental data showed the volumes of the two droplets were 16.6 and 20.8, respectively, and our numerical results predict 18.7 and 21.9, which are in agreement with the experimental results.
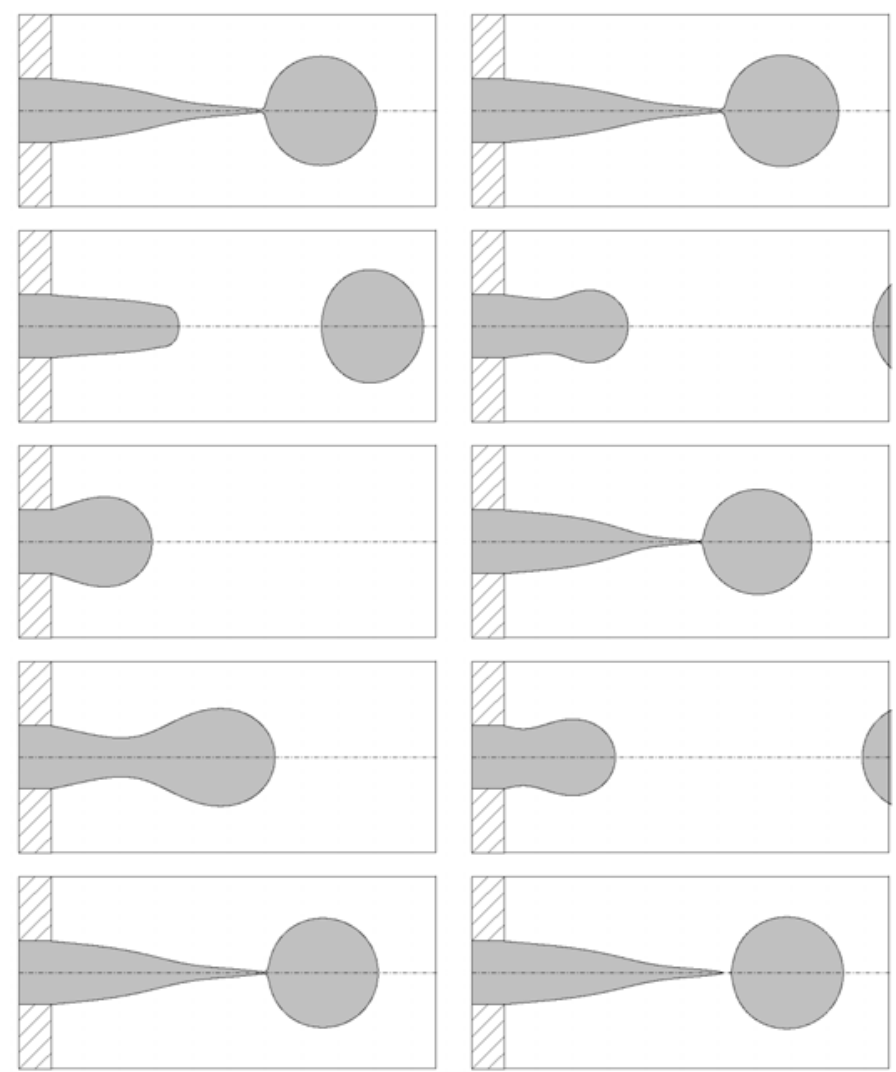

Fig. 8 Snapshots of interface shapes at $R e=4.34, B o=0.33$, and $\theta=120^{\circ}$ (left: $W e=0.16$ and from top to bottom, $t=1.09,1.10,1.12,1.17$, and 1.19, and right: $W e=0.2$ and from top to bottom, $t=0.81,0.82,0.89,0.90$, and 0.98$)$

\subsection{Drop impacting on rough substrate}

We consider axisymmetric simulations of drop impacting on a rough substrate. The flow phenomena of drop impacting on the smooth substrate have been extensively investigated, and a recent review can be found in Ref. [29]. We represent the rough substrate by a grooved substrate similar to the study of droplet motion inside a grooved channel by Huang et al. ${ }^{[30]}$. In the present work, we investigate the impact dynamics of a droplet on a grooved substrate (see Fig. 10). The diameter of the droplet is $D$, and the size of the computational domain is $1.1 D \times 2.5 D$ (the mesh $440 \times 1000)$. The grooves are of uniform width and depth, i.e., $0.08 D \times 0.05 D$, which also corresponds to the size of the blocks $a$ to $e$. There are totally ten blocks in the domain (see Fig. 10), and they can be classified into five groups according to their BCs. The $\mathrm{BCs}$ are $\left(\mathrm{BC}_{2}, \mathrm{BC}_{1}, \mathrm{BC}_{1}, \mathrm{BC}_{5}\right)$ for the block $a,\left(\mathrm{BC}_{1}, \mathrm{BC}_{1}, \mathrm{BC}_{1}, \mathrm{BC}_{5}\right)$ for the blocks $b$ to $e,\left(\mathrm{BC}_{2}, \mathrm{BC}_{6}, \mathrm{BC}_{5}, \mathrm{BC}_{4}\right)$ for the block $f,\left(\mathrm{BC}_{5}, \mathrm{BC}_{6}, \mathrm{BC}_{5}, \mathrm{BC}_{4}\right)$ for the blocks $g$ to $i$, and $\left(\mathrm{BC}_{5}, \mathrm{BC}_{6}, \mathrm{BC}_{4}, \mathrm{BC}_{4}\right)$ for the block $j$. The density and viscosity ratios of the air to the liquid are 0.001 and 0.025 , respectively. We choose the impact velocity of the droplet $V$ and $D$ as the characteristic velocity and length, respectively, and also use the properties of the liquid to define the dimensionless group. The time step $\Delta t=0.000025$. 


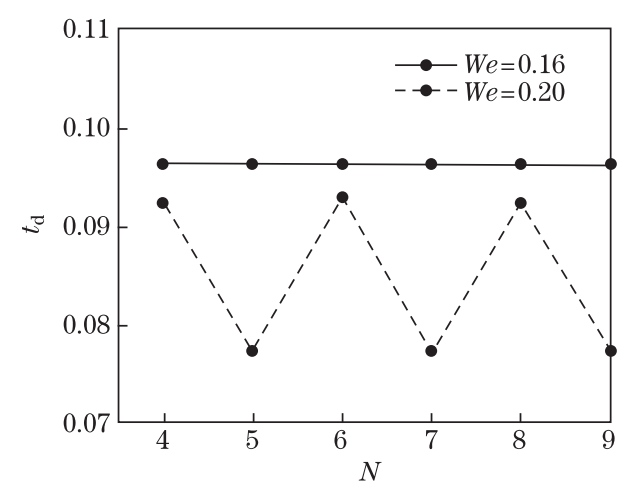

Fig. 9 Time interval between two successive drippings $\left(t_{\mathrm{d}}\right)$ as function of sequence number of drop $(N)$

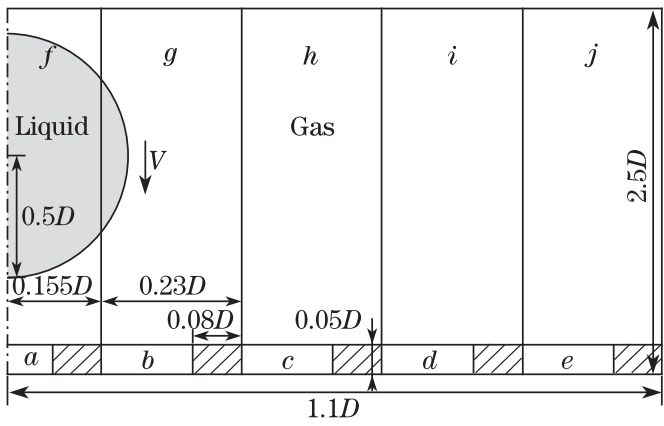

Fig. 10 Sketch of drop impacting on grooved substrate

We simulate two cases at $O h(=0.0035)$, i.e., Case $1: W e=12.5$ and $\theta=120^{\circ}$, and Case 2 : $W e=32$ and $\theta=140^{\circ}$. Figure 11 shows the snapshots of the droplet shapes at different time for the two cases. It is seen that in both cases, the droplet spreads on the substrate horizontally after the impact. However, the droplet in Case 2 also penetrates into the grooves and touches the bottom of some cavities during the spreading. In contrast, the droplet in Case 1 only forms the contact lines pinned at the corners of the grooves. As a result, the droplet in Case 1 rebounds from the substrate at the time $t=3.6$, while the droplet in Case 2 generates a satellite droplet ejected into the air, leaving the rest of the liquid attached to the substrate. It is found that the inertia of the droplet plays an important role in the impact dynamics, and may lead to the change of the wetting state on the rough substrate, e.g., partial transition from the Cassie state to the Wenzel state here ${ }^{[31]}$. It is also clear that the etching multiblock method can successfully capture the contact line pinned at the corners of the grooves, which has been observed in previous experiments ${ }^{[32]}$.

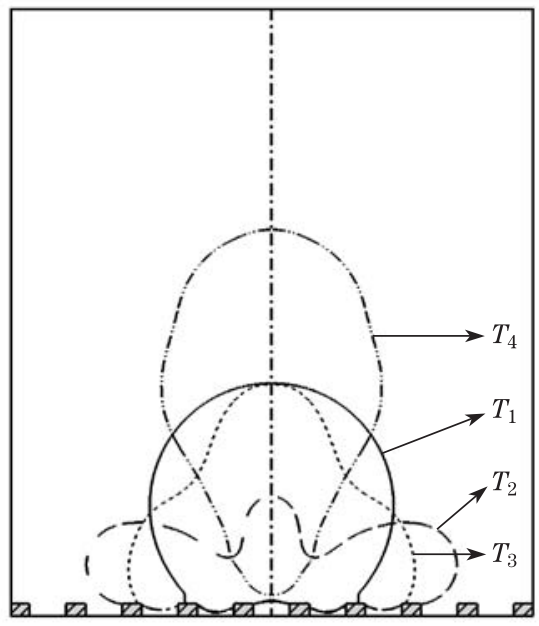

(a)

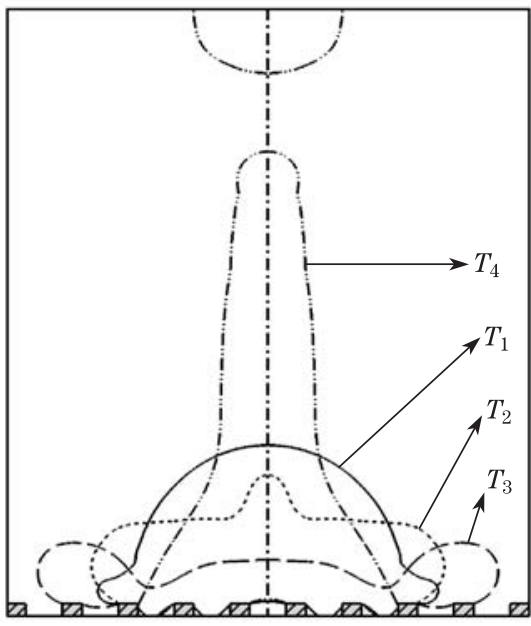

(b)

Fig. 11 Snapshots of drop impacting on grooved substrate with $O h=0.0035$, (a) We $=12.5$, $\theta=120^{\circ}$, and snapshot time $T_{1}=0.13, T_{2}=1.00, T_{3}=1.75$, and $T_{4}=3.60 ;(\mathrm{b}) W e=32$, $\theta=140^{\circ}$, and snapshot time $T_{1}=0.50, T_{2}=1.00, T_{3}=1.50$, and $T_{4}=3.13$ 


\subsection{Flow focusing}

The last application is the droplet generation by flow focusing, in which the liquid $\mathrm{A}$ in the capillary tube is driven by the liquid B through a pore (see Fig. 12). Flow focusing has been shown to be an effective way to generate uniform and monodispersed droplets ${ }^{[33]}$. However, because the geometry of the flow focusing device is rather complicated, and the flows involve two-phase flows and MCLs, there are only a few numerical studies of flow focusing phenomena, mainly using commercial softwares ${ }^{[34-36]}$.

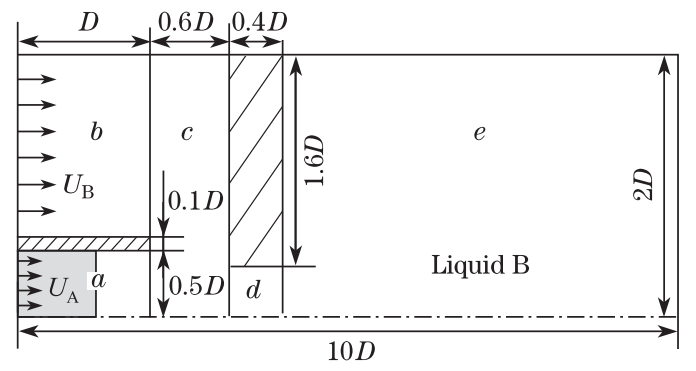

Fig. 12 Sketch of flow focusing device, where gray area shows initial space occupied by liquid A, and blank area is filled with liquid B

Figure 12 shows the numerical setup for axisymmetric simulations. The computational domain is $10 D \times 2 D$ (the mesh is $1000 \times 200$ ), where $D$ is the inner diameter of the capillary tube. There are five blocks $(a, b, c, d$, and $e)$, and the $\mathrm{BCs}$ of these blocks are $\left(\mathrm{BC}_{3}, \mathrm{BC}_{2}, \mathrm{BC}_{5}\right.$, $\left.\mathrm{BC}_{1}\right),\left(\mathrm{BC}_{3}, \mathrm{BC}_{1}, \mathrm{BC}_{5}, \mathrm{BC}_{1}\right),\left(\mathrm{BC}_{6}, \mathrm{BC}_{2}, \mathrm{BC}_{6}, \mathrm{BC}_{1}\right),\left(\mathrm{BC}_{5}, \mathrm{BC}_{2}, \mathrm{BC}_{5}, \mathrm{BC}_{1}\right)$ and $\left(\mathrm{BC}_{6}, \mathrm{BC}_{2}\right.$, $\left.\mathrm{BC}_{4}, \mathrm{BC}_{4}\right)$, respectively. We choose the average velocity at the inlet of the capillary tube $U_{\mathrm{A}}$ and $D$ as the characteristic velocity and length, respectively. The effect of gravity is considered to be negligible. The properties of the liquid A are used to define the dimensionless group: $R e$ and We. The density and viscosity ratios of the liquid $\mathrm{B}$ to the liquid $\mathrm{A}$ are 1.03 and 0.2 , respectively. The time step $\Delta t=0.000025$.

We investigate here two cases with $R e=1.7, W e=0.009$, and $\theta=120^{\circ}$ but different average velocities at the inlet of the fluid $\mathrm{B}$, particularly $U_{\mathrm{B}}=0.92$ and 1.26 , respectively. Figure 13 shows the numerical results of two cases in terms of the interface shape. It is interesting to see that the difference in the flow rate of the liquid B leads to the occurrence of different flow modes. The case with $U_{\mathrm{B}}=0.92$ appears to enter a dripping mode, in which droplets of uniform size are generated near the pore, and the cone of the liquid A oscillates periodically. The case with $U_{\mathrm{B}}=1.26$ appears to enter a jetting mode, in which a slender liquid jet is formed at the downstream of the pore, and the shape of the liquid cone is kept unchanged with the time. The second case also continuously generates droplets, which are smaller than those in the first case. We note that the dripping and jetting modes are in consistence with the experimental observations $^{[37]}$.

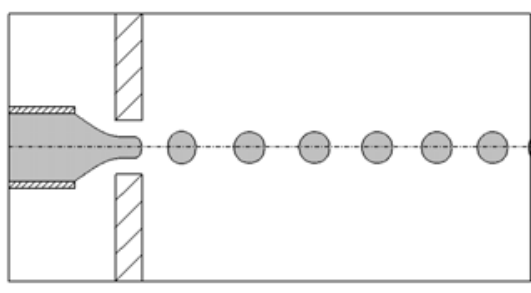

(a)

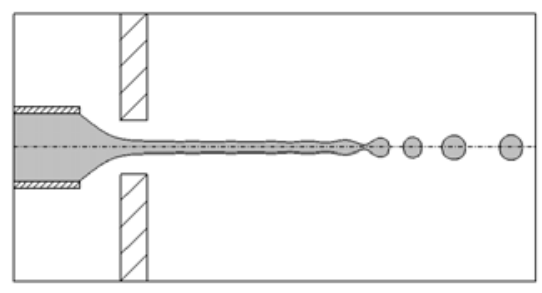

(b)

Fig. 13 Dripping and jetting modes in droplet generation by flow focusing device, (a) $U_{\mathrm{B}}=0.92$ (dripping) and (b) $U_{\mathrm{B}}=1.26$ (jetting) 


\section{Conclusions}

We combine the etching multiblock method with the DI method and the geometric formulation of MCL model to simulate incompressible two-phase flows in the presence of a complex geometry. The complex geometry can be decomposed into multiple blocks of regular shapes such as rectangles and squares. One layer of ghost cells is placed around each block, for the convenience of implementing the BCs. The BCs include the physical ones, such as the MCL model for the volume fraction, and those for the connections between the blocks. The latter shall require no treatment at all. As a result, the etching multiblock method can be extended from the single-phase flows to the multiphase flows in a straightforward manner. The etching multiblock method is shown to be able to effectively deal with the incompressible two-phase flows in a domain with multiple blocks. Its performance is examined through a few numerical experiments. The simulation of single-phase flow past a square cylinder shows that the results converge with the mesh refinement. The numerical results of drops dripping from a pore are in good agreement with the experimental results qualitatively and quantitatively. Finally, we use the method to the droplet generation in flow focusing and drop impacting on the rough substrate.

Acknowledgements The authors acknowledge the support of the 100 Talents Program of the Chinese Academy of Sciences.

\section{References}

[1] Hu, D. L., Chan, B., and Bush, J. W. M. The hydrodynamics of water strider locomotion. nature, 424, 663-666 (2003)

[2] Vella, D. Floating versus sinking. Annu. Rev. Fluid Mech., 47, 115-135 (2015)

[3] Gao, P. and Feng, J. J. A numerical investigation of the propulsion of water walkers. J. Fluid Mech., 668, 363-383 (2011)

[4] Fan, E. S. C. and Bussmann, M. Piecewise linear volume tracking in spherical coordinates. Appl. Math. Model., 37, 3077-3092 (2013)

[5] Uhlmann, M. An immersed boundary method with direct forcing for the simulation of particulate flows. J. Comput. Phys., 209, 448-476 (2005)

[6] Mittal, R. and Iaccarino, G. Immersed boundary methods. Annu. Rev. Fluid Mech., 37, 239-261 (2005)

[7] Mittal, R., Dong, H., Bozkurttas, M., Najjar, F. M., Vargas, A., and von Loebbecke, A. A versatile sharp interface immersed boundary method for incompressible flows with complex boundaries. $J$. Comput. Phys., 227, 4825-4852 (2008)

[8] Ren, W. W., Shu, C., Wu, J., and Yang, W. M. Boundary condition-enforced immersed boundary method for thermal flow problems with Dirichlet temperature condition and its applications. Comput. Fluids, 57, 40-51 (2012)

[9] Ding, H., Spelt, P. D. M., and Shu, C. Diffuse interface model for incompressible two-phase flows with large density ratios. J. Comput. Phys., 226, 2078-2095 (2007)

[10] Liu, H. R. and Ding, H. A diffuse-interface immersed-boundary method for two-dimensional simulation of flows with moving contact lines on curved substrates. J. Comput. Phys., 294, 484-502 (2015)

[11] Wan, D. C., Patnaik, B. S. V., and Wei, G. W. Discrete singular convolutionfinite subdomain method for the solution of incompressible viscous flows. J. Comput. Phys., 180, 229-255 (2002)

[12] Raspo, I. A direct spectral domain decomposition method for the computation of rotating flows in a T-shape geometry. Comput. Fluids, 32, 431-456 (2003)

[13] Abide, S. and Viazzo, S. A 2D compact fourth-order projection decomposition method. J. Comput. Phys., 206, 252-276 (2005)

[14] Jacqmin, D. Calculation of two-phase Navier-Stokes flows using phase-field modeling. J. Comput. Phys., 155, 96-127 (1999) 
[15] Biben, T., Kassner, K., and Misbah, C. Phase-field approach to three-dimensional vesicle dynamics. Phys. Rev. E, 72, 041921 (2005)

[16] Folch, R., Casademunt, J., Hernandez-Machado, A., and Ramirez-Piscina, L. Phase-field model for Hele-Shaw flows with arbitrary viscosity contrast I: theoretical approach. Phys. Rev. E, 60, 1724-1733 (1999)

[17] Yue, P. T., Feng, J. J., Liu, C., and Shen, J. A diffuse-interface method for simulating two-phase flows of complex fluids. J. Fluid Mech., 515, 293-317 (2004)

[18] Ding, H. and Spelt, P. D. M. Wetting condition in diffuse interface simulations of contact line motion. Phys. Rev. E, 75, 046708 (2007)

[19] Ding, H. and Spelt, P. D. M. Inertial effects in droplet spreading: a comparison between diffuseinterface and level-set simulations. J. Fluid Mech., 576, 287-296 (2007)

[20] Ding, H. and Spelt, P. D. M. Onset of motion of a three-dimensional droplet on a wall in shear flow at moderate Reynolds numbers. J. Fluid Mech., 599, 341-362 (2008)

[21] Ding, H., Gilani, M. N. H., and Spelt, P. D. M. Sliding, pinch-off and detachment of a droplet on a wall in shear flow. J. Fluid Mech., 644, 217-244 (2010)

[22] Ding, H., Li, E. Q., Zhang, F. H., Sui, Y., Spelt, P. D. M., and Thoroddsen, S. T. Propagation of capillary waves and ejection of small droplets in rapid droplet spreading. J. Fluid Mech., 697, 92-114 (2012)

[23] Breuer, M., Bernsdorf, J., Zeiser, T., and Durst, F. Accurate computations of the laminar flow past a square cylinder based on two different methods: lattice-Boltzmann and finite-volume. Int. J. Heat and Fluid Flow, 21, 186-196 (2000)

[24] Okajima, A. Strouhal numbers of rectangular cylinders. J. Fluid Mech., 123, 379-398 (1982)

[25] Sumesh, P. T. and Govindarajan, R. The possible equilibrium shapes of static pendant drops. $J$. Chem. Phys., 133, 144707 (2010)

[26] Sui, Y., Ding, H., and Spelt, P. D. M. Numerical simulations of flows with moving contact lines. Annu. Rev. Fluid Mech., 46, 97-119 (2014)

[27] Ding, H., Chen, B. Q., Liu, H. R., Zhang, C. Y., Gao, P., and Lu, X. Y. On the contact-line pinning in cavity formation during solid-liquid impact. J. Fluid Mech., 783, 504-525 (2015)

[28] Subramani, H. J., Yeoh, H. K., Suryo, R., Xu, Q., Ambravaneswaran, B., and Basaran, O. A. Simplicity and complexity in a dripping faucet. Phys. Fluids, 18, 032106 (2006)

[29] Yarin, A. L. Drop impact dynamics: splashing, spreading, receding, bouncing. Annu. Rev. Fluid Mech., 38, 159-192 (2006)

[30] Huang, J. J., Shu, C., and Chew, Y. T. Lattice Boltzmann study of droplet motion inside a grooved channel. Phys. Fluids, 21, 022103 (2009)

[31] Wenzel, R. N. Resistance of solid surfaces to wetting by water. Ind. Eng. Chem., 28, 988-994 (1936)

[32] Cassie, A. B. D. and Baxter, S. Wettability of porous surfaces. Trans. Faraday Soc., 40, 546-550 (1944)

[33] Barrero, A. and Loscertales, I. G. Micro- and nano-particles via capillary flows. Annu. Rev. Fluid Mech., 39, 89-106 (2007)

[34] Herrada, M. A., Ganan-Calvo, A. M., Ojeda-Monge, A., Bluth, B., and Riesco-Chueca, P. Liquid flow focused by a gas: jetting, dripping, and recirculation. Phys. Rev. E, 78, 036323 (2008)

[35] Vega, E. J., Montanero, J. M., Herrada, M. A., and Ganan-Calvo, A. M. Global and local instability of flow focusing: the influence of the geometry. Phys. Fluids, 22, 064105 (2010)

[36] Montanero, J. M., Rebollo-Munoz, N., Herrada, M. A., and Ganan-Calvo, A. M. Global stability of the focusing effect of fluid jet flows. Phys. Rev. E, 83, 036309 (2011)

[37] Gañán-Calvo, A. M. and Riesco-Chueca, P. Jetting-dripping transition of a liquid jet in a lower viscosity co-flowing immiscible liquid: the minimum flow rate in flow focusing. J. Fluid Mech., 553, 75-84 (2006) 